\title{
Bildunsphilosophie und empirische Forschung
}

\author{
Filosofia da educação e pesquisa empírica
}

Werner Thole*

\section{Zusammenfassung}

Die erziehungs- und sozialwissenschaftliche Forschungslandschaft hat sich in den zurückliegenden Jahrzehnten weltweit enorm expandiert und ausdifferenziert. Befördert wird diese Entwicklung durch die internationalen Vergleichsstudien derOECD wie das“Programme for International Student Assessment" oder die Studie „Education at a Glance 2016". Zu registrieren ist nicht nur ein deutlicher Zuwachs an empirischen Forschungsvorhaben, sondern auch und insbesondere eine Weiterentwicklung der methodologischen Konzepte, Standards und Ansprüche. In dem Beitrag wird aus einer erziehungswissenschaftlichen Perspektive der Zuwachs an empirischer Forschung zu zentralen Fragen der Bildung erörtert. Nach einer Vergewisserung, was heute gemeint sein kann, wenn über Bildung gesprochen wird, und einem knappen Rückblick auf die Geschichte der pädagogischen Forschung wird erörtert, worin der Ertrag einer empirischen Beobachtung von Bildungsprozessen liegen kann. Diskutiert wird in diesem Zusammenhang auch die Ambivalenz zwischen quantitativ-statistischen und qualitativ-verstehenden Forschungsansätzen im Kontext einer erziehungswissenschaftlichen Forschung. In Erinnerung an die Beiträge Theodor W. Adornos zur empirischen Forschung wird dafür plädiert, den Streit über die Angemessenheit und Relevanz von unterschiedlichen Methoden nicht mehr theoriedogmatisch, sondern unter Bezug auf die jeweils in den Blick genommenen Fragestellungen zu führen. Gewarnt wird in diesem Kontext auch davor, die empirische Forschung gegen eine philosophisch orientierte Erziehungswissenschaft zu positionieren oder die kritische Bildungsphilosophie gegen empirisch grundierte Erkenntnisintentionen. Forschung bedarf der Theorie wie andererseits eine solide Generierung von theoretischem Wissen auf empirische Daten nicht verzichten sollte.

Schlüsselwörter: Empirie und Forschung. Kritische Theorie. Pädagogische Forschung. Pädagogische Theorie.

\section{Resumo}

O panorama da pesquisa educacional e social científica tem se expandido e se diferenciado enormemente nas últimas décadas, no mundo todo. Esse desenvolvimento é orientado por estudos internacionais de comparação da Organization for European Economic Co-operation (OECD), como o Programa para Aceitação Internacional de Estudante ou o estudo Education at a Glance 2016. Cabe destaque, nesse contexto, não só para o crescimento claro nos projetos de pesquisa empírica, mas também, e principalmente, para o desenvolvimento aprofundado de conceitos, estandartes e pretensões metodológicas. Nesta contribuição, será discutido, por meio da perspectiva educacional-científica, o crescimento da pesquisa empírica como questão central da ideia de formação. Segundo determinada certificação, o que pode, hoje, ser pensado quando se fala sobre formação e o que é discutido mediante um olhar abreviado sobre a história da pesquisa pedagógica auxiliam a perceber em que repousa a contribuição da observação empírica em relação ao processo de formação. Também será discutida,

Recebido em: 22/04/2017 - Aprovado em: 02/08/2017

http://dx.doi.org/10.5335/rep.v24i3. 7759

Promotion in Philosophie an der Universität Dortmund und Professor an der Universität Kassel. Kontakt: wthole@uni-kassel.de 


\begin{abstract}
no contexto da pesquisa educacional-científica, a ambivalência entre princípios de pesquisa quantitativo-estatísticos e qualitativo-interpretativos. Será defendido, em lembrança às contribuições de Theodor W. Adorno sobre pesquisa empírica, que a disputa sobre adequabilidade e relevância de diferentes métodos não repousa simplesmente no âmbito teórico dogmático, mas também se refere aos próprios problemas de investigação que aparecem no momento mesmo da pesquisa. Também será advertido como errôneo, nesse contexto, querer posicionar a pesquisa empírica contra a ciência da educação orientada filosoficamente ou a filosofia crítica da educação contra intenções epistemológicas graduadas empiricamente, pois a pesquisa precisa da teoria tanto quanto, de outro modo, a sólida generalização do conhecimento teórico não pode renunciar a dados empíricos.
\end{abstract}

Palavras-chave: Empiria e pesquisa. Pesquisa pedagógica. Teoria crítica. Teoria pedagógica.

Welches Wissen die Menschheit benötigt, um ihre Zukunft zu gestalten, ist weltweit gegenwärtig wohl die prominenteste Frage aufden Agenden der politischen und gesellschaftlichen Diskussionsforen. Lernen, Erziehung und Bildung avancierten zu zentralen Begriffen eines öffentlichen, globalisierten Diskurses. Hoffnungsvoll ist zu hören, dass mit einer Erweiterung der Möglichkeiten, Bildung zu erwerben, die Existenz der regionalen, länderspezifischen wie weltweiten sozialen Ungleichheiten beseitigt werden kann. Soziale Gerechtigkeit scheint möglich. Inwiefern diese Hoffnung, mittels Bildung soziale Ungleichheiten zu beseitigen, berechtigt ist, bleibt gleichwohl unsicher. Die zu beobachtende Verteilung des ökonomischen Kapitals lässt eher vermuten, dass das „Elend der Welt" und Leiden vieler Menschen an der Gesellschaft (vgl. BOURDIEU, 1993) mittels Bildung nicht vermindert werden kann. Die gegebenen ökonomischen und politischen Machtstrukturen scheinen resistent zu sein, trotz der Erweiterung von Bildungsmöglichkeiten.

In vielen Ländern hat sich angesichts des Bedeutungsgewinns von Bildung die pädagogische, erziehungs- und sozialwissenschaftliche Forschungslandschaft in den zurückliegenden Jahrzehnten weltweit und insbesondere auch in der Bundesrepublik Deutschland enorm entwickelt. Die internationalen Vergleichsstudien der OECD wie das "Programme for International Student Assessment" oder die Studie "Education at a Glance 2016" sind ein Ausdruck dieser Entwicklung. Die Diskussion von Forschungsbefunden scheint partiell sogar die Erörterung von Theorien zu verdrängen. $\mathrm{Zu}$ registrieren ist jedoch nicht nur ein deutlicher Zuwachs an Forschungsvorhaben, sondern auch und insbesondere eine Weiterentwicklung der methodologischen Konzepte sowie eine Spezifizierung der forschungsmethodischen Verfahren. Die Entwicklung betrifft nicht nur das quantitativ-heuristische Forschungsspektrum, das sich im Zuge der Ausbreitung und Etablierung der internationalen Vergleichsstudien im Bildungsund Sozialbereich methodisch ausdifferenzierte. In besonderem Maße trifft diese Markierung auch auf die qualitativ-rekonstruktiven Forschungsansätze 
in der Erziehungswissenschaft und Soziologie zu. Sie verloren inzwischen ihren ehemaligen alternativen und exotischen Charakter und die ursprünglich eher deskriptiven Analyse- und Auswertungskonzepte qualifizierten sich zu elaborierten methodologischen Ansätzen und methodischen Zugängen.

Nach einer Verständigung über das, was der deutsche Begriff Bildung meint und wo er von Erziehung, Sozialisation und Lernen zu unterscheiden ist (1), wird nachfolgend in dem Aufsatz daran erinnert, dass es neben einem philosophischen seit dem frühen zwanzigsten Jahrhundert einen empirisch fundierten Diskurs zu pädagogischen Fragen gibt (2). Anknüpfend an diese Überlegungen wird dafür plädiert, der empirischen, pädagogischen Bildungsforschung nicht nur die Forschungsvorhaben zuzurechnen, die auf quantitativ-heuristische Verfahren zurückgreifen, sondern auch diejenigen, die Fragestellungen unter Rückgriff auf qualitativ-rekonstruktive Methoden aufzuklären versuchen (3). Abschließend wird dafür plädiert, den Streit über die Angemessenheit und Relevanz von quantitativ-heuristischen und qualitativ-rekonstruktiven Methoden nicht mehr theoriedogmatisch, sondern unter Bezug auf die jeweils in den Blick genommenen Fragestellungen $\mathrm{zu}$ führen (4). Die Ausführungen sind fokussiert auf den deutschsprachigen Diskurs. Die in dem Beitrag erörterten Fragen spiegeln jedoch exemplarisch ähnliche Diskussionen in anderen Sprachräumen und überschreiten so durchaus den engmaschigen deutschen Sprachzaun.

\section{Zum Begriff der Bildung}

Bildung ist sicherlich einer der zentralen, wenn nicht sogar der zentrale und historisch bedeutsamste Begriff pädagogischen und erziehungswissenschaftlichen Denkens, auch wenn er in englisch-, spanisch-und portugiesischen Diskursen eher eine randständige Bedeutung hat. Die mit dem Bildungsbegriff aufgerufenen Intentionen finden sich bereits in der altgriechischen Philosophie und in Texten des Hoch- und Spätmittelalters ebenso wie in Abhandlungen von Autoren der französischen und deutschen Aufklärung, des Neuhumanismus und der frühen Kapitalismuskritik notiert. Ausgehend von der Annahme, dass der Mensch zu Beginn seiner Entwicklung noch "unausgestattet« (askometon) ist, seine Körperlichkeit und -seele sich zwar natürlich entwickelt, nicht jedoch seine zweite, gesellschaftliche Natur, die Denk- und Vernunftseele, begründet sich die frühe Idee der Erziehung, Bildung und Bildsamkeit als »paideia «. Auf dieser Vorstellung der Zweiteilung der menschlichen Onto- wie Phylogenese basiert im Kern die Geschichte der Idee von Bildung und Bildsamkeit und letztendlich auch die der Formbarkeit und der Selbstbestimmungsfähigkeit des Menschen (vgl. BENNER; BRÜGGEN, 2004; ZIRFAS, 2010). 
In der christlich religiösen, abendländischen Tradition verweist der Bildungsbegriff auf eine Metapher aus der Genesispassage im ersten Buch Moses der Bibel (vgl. STOJANOV, 2006) - Gott sprach: "Lasset uns Menschen machen, ein Bild (tselem), das uns gleich sei, die da herrschen über die Fische im Meer und über die Vögel unter dem Himmel und über das Vieh und über alle Tiere des Feldes und über alles Gewürm, das auf Erden kriecht" (MOSE, K. 1, v. 26). Gott hat der hier mitgeteilten Vorstellung zufolge den Menschen nach seinem Bilde geschaffen - mit anderen Worten: sich nachgebildet -, zugleich aber den nachgebildeten Menschen verboten, sich ein Bild Gottes zu machen. Dieser religiös-mystizistische Bedeutungsgehalt findet sich auch in dem im 14. Jahrhundert durch den Theologen und Philosophen Eckhart von Hochheim, bekannt als Meister Eckhart, in die deutsche Sprache eingeführten Bildungsbegriff. Für ihn bedeutet Bildung das »Erlernen von Gelassenheit« und die Schaffung eines gottähnlichen Menschen. Das Wort Bildung in seiner heutigen Verwendung entstammt dem althochdeutschen Begriff »bildunga «, mittelhochdeutsch »bildunge«, und meint Schöpfung, Bildnis und Gestaltung, bezeichnet also die Formung des Menschen im Hinblick auf sein »Menschsein«.

Die hier nur angedeutete, lange Geschichte des Begriffs Bildung wird allerdings in pädagogischen Diskursen zuweilen ignoriert und auf die zurückliegenden Jahrzehnte verkürzt oder aber als ein Erbe der geisteswissenschaftlichen Pädagogik vorgestellt. Insbesondere in der Kritik an der geisteswissenschaftlichen Pädagogik erfährt der Begriff Bildung derart vielfältige semantische Fassungen, dass sogar dafür plädiert wird, den Bildungsbegriff, »im Gegensatz zu Begriffen wie Lernen oder Sozialisation, nicht präzise zu definieren, sondern nur zu dimensionieren" (EHRENSPECK, 2004, S. 66; vgl. ZIRFAS, 2010).

Unter Bildung ist zunächst nichts anderes zu verstehen als der individuelle Erwerb von Fähigkeiten auf der Basis von reflektierten Erfahrungen, die es dem Subjekt ermöglichen, sich selbst in den sozialen, gesellschaftlichen Welten $\mathrm{zu}$ verorten und zudem und darüber hinaus sich die Welt zu erklären und zu erschließen. Der Erwerb von Welterkenntnissen setzt die Aneignung von Bildung und die Realisierung von Bildungsprozessen ebenso voraus wie die Erlangung von Subjektivität, Personalität und Identität sowie die Entwicklung von Wissen und Gefühlen über die eigene Selbstwirksamkeit. Diese Bestimmung scheint zunächst ausreichend, um das, was Bildung meint, von dem zu differenzieren, was unter Sozialisation und Erziehung zu verstehen ist. Sozialisation beschreibt und meint im Kontrast zur Bildung die gesamten, gesellschaftlich vermittelten, von der sozialen und materiellen Umwelt gerahmten individuellen Entwicklungsprozesse der Entstehung von Persönlichkeit, Individualität und Identität, Eigenständigkeit und 
der Herstellung von sozialer Integration. Beschreibt Sozialisation die Gesamtheit der Bedingungen und Formen der Herstellung von Gesellschaftsfähigkeit, bezieht sich Erziehung auf die Benennung von Entwicklungspraxen, die sich über ausgewiesene, direkte, beispielsweise pädagogische Interventionen, oder indirekte Einflüsse, beispielsweise medial initiierte Prozesse, konstituieren. Seit Jahrzehnten wird Erziehung zudem als »die Summe der Reaktionen einer Gesellschaft auf die Entwicklungstatsache«(BERNFELD, 1973, S. 57) definiert. Die Initiierung von Erziehung basiert demnach auf die Markierung einer generationalen Differenz, also auf den Umstand, dass junge Menschen, unabhängig davon, in welchen Kulturen und unter welchen Verhältnissen sie aufwachsen, nicht qua Geburt mit den gleichen sozialen, emotionalen, kommunikativen und kognitiven Kompetenzen ausgestattet sind wie ältere. Diese Begründungsfolie für Erziehung erweist sich jedoch zunehmend als empirisch wenig belastbar, da beispielsweise die Verfügbarkeit über Wissen heute keineswegs mehr ein ausschließliches Privileg der jeweils älteren Generation ist (vgl. THOLE, 2008). Demgegenüber bezeichnet Lernen einen Prozess der Verhaltensänderung durch den Erwerb von Wissen und Können, also von kognitiven, sozialen und kulturellen Kenntnissen und Fertigkeiten. Unter einer entwicklungs- und lernpsychologischen Perspektive wird das Gedächtnis als der zentrale Speicherort des Gelernten markiert. Neben dem klassischen, konditionierenden Lernen wird in den psychologischen Diskursen unter anderem zwischen dem operationalen respektive instrumentellen Lernen, dem Diskriminationslernen, dem kognitiven Lernen und dem sozialen Lernen unterschieden. In der pädagogischen und erziehungswissenschaftlichen Diskussion ist allerdings eine Skepsis gegenüber den genannten theoretischen Modellen wahrzunehmen. Ein über die Modelle konzipiertes Lernen hat sich unter anderem - neben dem Technologievorwurf und der darüber angeregten Kritik - damit auseinanderzusetzen, dass unter experimentellen Bedingungen validierte Formen des Lernens sich nicht in den pädagogischen Alltagsszenarien in vergleichbarer Form reproduzieren. Insbesondere die Forschung stößt auf Schwierigkeiten, das artikulierte Wissen und Können nachweislich und belastbar auf konkrete Methoden des Lehrens und Lernens zu beziehen. Konsens besteht weitgehend darin, dass sich die Formen der Erziehung ebenso wie die der Sozialisation, der Bildung und des Lernens in den zurückliegenden drei Dekaden dynamisierten und veränderten.

Die Hinweise verdeutlichen, dass über Bildung reden immer auch bedeutet, über Sozialisation, Erziehung und Lernen nachzudenken. Auch über die Hinweise wird deutlich, dass es kompliziert ist, eine allgemein gültige, von unterschiedlichen theoretischen Perspektiven getragene Bestimmung von Bildung zu präsentieren. 
Bildung in der geisteswissenschaftlichen Tradition fokussiert beispielsweise auf die Entwicklung der Selbst- und Welterkenntnisfähigkeiten, die marxistische und die emanzipatorisch gefärbte Programmatik von Bildung setzt auf die Idee der individuellen Selbstbestimmung und die Veränderung der ungerechten gesellschaftlichen Verhältnisse, die jüngere empirische Bildungsforschung denkt Bildung als eine empirisch operationalisierbare Kompetenzdimension, eine humanistische Bildungsidee denkt Bildung als Herausbildung von individuellen Gestaltungsfähigkeiten und die ästhetische oder phänomenologische Idee adressiert an Bildung die Entwicklung von Empathie und Sinnlichkeit (vgl. u. a. ZIRFAS, 2010). Im Kern stimmen diese programmatischen Fassungen des Bildungsbegriffs zumindest dahingehend überein, dass Bildung beinhaltet, über gelungene Formen der Selbstverortung und der Welterkenntnis zu verfügen. Dies beinhaltet die Fähigkeiten, Traditionen und soziale und kulturelle Überlieferungen befragen zu können, über Empathie und eine generationale Lokalisierungskompetenz zu verfügen und das Vermögen, ein Leben in sozialer Gemeinschaft zu bewältigen, zu verstehen und letztendlich auch zu gestalten (vgl. u. a. TIPPELT, 1990).

Werden inhaltlich-strukturelle Aspekte der Markierung von unterschiedlichen Bildungsbegriffen und -definitionen herangezogen, dann kann zwischen einer formalen, einer materiellen und einer kategorialen Idee und Konzeption von Bildung unterschieden werden:

- Die Theorie einer formalen Bildung versteht die gesellschaftlich vorgehaltenen Wissenskontexte als Ressourcen für die Entwicklung von Praxen der subjektiven Aneignung von Fähigkeiten und Fertigkeiten, Qualifikationen und Kompetenzen. Eine so akzentuierte Idee von Bildung findet sich zuweilen verkürzt auf die Erlangung von formalen Abschlüssen und Zertifikaten. Doch auch wenn gegenüber einer derartigen Verkürzung Distanz besteht, reduziert eine über formale Inhalte ausbuchstabierte Bildungstheorie die Idee einer allgemeinen Bildung auf das Wissen und Können, das gesellschaftlich anerkannt und legitimiert ist.

- Gegenüber dieser Konzeption beschreibt eine materiale Bildungstheorie präzise die Inhalte und Wissensbereiche, die von den Subjekten im Verlauf ihrer Bildungsbiografie anzueignen sind. Bildung ist demnach die Aneignung eines mehr oder weniger eindeutig kanonisierten Fundus von Wissen und vielleicht noch von gesellschaftlichen Erfahrungen. Alle auf materiale Inhalte basierenden Bildungstheorien, so wird kritisch formuliert, stimmen darin überein, 
[...] so sehr sie sich untereinander auch unterscheiden mögen [...], dass sie zwischen den gesellschaftlichen Anforderungen an die pädagogische Praxis [...] und der pädagogischen Praxis eine teleologische Verhältnisbestimmung konstruieren und der pädagogischen Praxis die Aufgabe zuweisen, die Heranwachsenden im Sinne vorgegebener gesellschaftlicher Anforderungen zu handlungsfähigen Mitgliedern der Gesellschaft zu machen (BENNER, 1987, S. 126).

- Im Konsens mit dieser Kritik und den zuletzt notierten Einwurf aufgreifend gehen kategoriale Bildungstheorien von einer Dialektik zwischen Ich und Welt, der Aneignung von Wissen und der Kritik der Inhalte sowie zwischen Wissen und Können aus. Um entsprechende, dialektisch modellierte Bildungsprozesse anregen zu können, bedarf es allerdings einer »relativen Selbstständigkeit pädagogischer Institutionen«, denn nur dann, »in einer gewissen Distanz zu ökonomischen und gesellschaftlichen Verhältnissen«, kann Bildung »Aufklärungsprozesse anregen, kritisches Bewusstsein [...] vermitteln und Perspektiven der Veränderung [...] eröffnen« (KLAFKI; BRAUN, 2007, S. 73). Im Kontrast zu formalen und materialen Bildungstheorien, denen ihr affirmativer Grundtenor auch von Vertretern kategorialer Bildungstheorien vorgehalten wird, reklamieren kategoriale Theorien eine nicht affirmative Konzeption von Bildung (vgl. BENNER; BRÜGGEN, 2004). Nicht affirmative, kritische Theorien der Bildung betonen zudem, dass Modelle von Bildung, die einzig darauf orientieren, die gegebene Gesellschaft und das in dieser vorrätig gehaltene Wissen $\mathrm{zu}$ reproduzieren, also nicht weniger intendieren und ermöglichen als »Halbbildung« (vgl. ADORNO, 1973).

Bildung, die nicht affirmativ ausgerichtet ist, die also darauf abzielt, Subjekten das Durchschauen gesellschaftlicher Wirklichkeit zu ermöglichen sowie sich selbst in der Welt zu lokalisieren, basiert auf der Idee von Mündigkeit. Bildung in diesem Sinne ist zu verstehen als »Aktualisierung der Potenzialität«, dass der Mensch »sein eigener Täter (HEYDORN, 1995, S. 138) sein kann, also optional gewillt und in der Verfassung ist, Wirklichkeit nicht nur zu erleben und zu ertragen, sondern auch zu gestalten. Bildung platziert sich so als Antipode zur bürgerlich-kapitalistischen Gesellschaft, thematisiert die Herrschaftsförmigkeit von Bildung als ein Problem der Okkupation von Wissen zur Stabilisierung und Legitimierung ungerechter gesellschaftlicher Strukturen. In dieser radikal kritischen Modellierung der Bildungsidee verliert sich die Ambiguität zwischen Gesellschaft und Individuum und »ihre klassengeschichtliche Zerrissenheit« (HEYDORN, 1995, S. 293) wird überwunden. Für die Analyse sozialer Ungleichheiten sensible Theorien vertiefen diese Idee von Bildung empirisch über die Analyse der Bildungs- und Sozialsysteme, 
die als Orte der Reproduktion der gesellschaftlichen Kräfteverhältnisse strukturell wie symbolisch identifiziert werden. Bildung wird in dieser Perspektive als das Resultat der gegebenen und erlebten gesellschaftlichen Verhältnisse, als die zur Natur gewordene Spiegelung von Wissen, Traditionen und Erfahrungen, Urteilsund Bewertungsschemata entworfen (vgl. BOURDIEU, 1985, S. 1987). Im Habitus manifestieren sich die subjektiv angeeigneten, aber gesellschaftlich gerahmten Formen des Denkens.

Unter einer spezifischen bildungstheoretischen Perspektive scheint nicht nur, aber insbesondere dem kulturellen Kapital eine besondere Aufmerksamkeit zuzukommen. Pierre Bourdieu (1985) unterscheidet zwischen dem inkorporierten, objektivierten und institutionalisierten kulturellen Kapital. Inkorporiertes Kulturkapital meint Bildung, welche langfristig in Erziehungs- und Bildungseinrichtungen sowie innerhalb der Familie als Wissen erworben wird. Objektiviertes Kulturkapital beschreibt den Besitz von kulturellen, ästhetischen, musikalischen und literarischen Gütern. Objektiviertes Kulturkapital gewinnt allerdings erst im Zusammenhang mit dem inkorporierten Kulturkapital Bedeutsamkeit, da der Gebrauch der Objekte Bildung voraussetzt. Institutionalisiertes Kulturkapital meint Titel und Bildungspatente, die dauerhafte Gültigkeit und damit einen gesellschaftlich und rechtlich anerkannten Wert besitzen. Unter soziales Kapital fasst P. Bourdieu die Ressourcen, die auf der Zugehörigkeit zu einer Gemeinschaft, also soziale Netzwerke und Beziehungen verweisen. Die Konstitution des Habitus erfolgt demzufolge nie losgelöst von den gesellschaftlich präjudizierten, traditionellen, milieuspezifisch und familial geprägten und in die Praxen. Über die aktivierenden sozialen Praktiken bleibt Bildung respektive der Habitus aber lebenslang gestaltbar. Formatierungen und Neuformatierungen schreiben sich dann wiederum in die Körper wie in die sozialen Praktiken ein und ermöglichen Neuformatierungen des Habitus. Bildung wie Habitus sind somit Resultate eines lebenslangen Prozesses und Ausdrücke von performativen gesellschaftlichen Praxen. Habitus - wie auch Bildung - bezeichnet die Verknüpfung von Kultur und Individualität sowie von Gesellschaft und Subjekt. Bildung ermöglicht den Menschen unter Bezug auf die ihnen jeweils zugänglichen oder von ihnen angeeigneten ökonomischen, sozialen und insbesondere kulturellen Kapitalressourcen an der Gestaltung ihrer Selbst- und Weltverhältnisse mündig und kritisch mitzuwirken. 


\section{Sammeln, Beobachten, Verstehen - Blick zurück}

Die historische Betrachtung regt dazu an, die Genese der pädagogischen Forschung in der Frühphase des 20. Jahrhunderts anzusiedeln. Wird eine Rekonstruktion der empirischen Sozialforschung anhand früher Studien unternommen, dann votieren gute Hinweise sogar dafür, davon auszugehen, dass eine qualitativ-deskriptive sich sogar vor der quantitativ-statistischen Wirklichkeitssicht herausbildet.

In den ersten Jahrzehnten der pädagogischen Forschung dominierten beobachtende, feld- und lebensweltbezogenen Methoden zusammen mit anderen qualitativ-rekonstruktiven Methoden wie Interviewverfahren, Spontanberichten, Tagebuch- und Aufsatzmethoden gegenüber rein statistischen Verfahren die pädagogisch motivierten Forschungsbemühungen. Begleitet wurde die Vorlage von kleineren Studien von Plädoyers, die Forschungsbemühungen zu intensivieren. So führtbeispielsweise Theodor Fritzsch (1906, S. 947)in der »ZeitschriftfürPhilosophie und Pädagogik « aus, dass die »unzeitige Theoriesucht der Sterblichen [...] eins der mächtigen Hindernisse « darstellt, »die den Fortgang der menschlichen Erkenntnis hemmen. Um der Pädagogik mehr Vollkommenheit zu verschaffen, muss sie auf Erfahrung gegründet werden. Dazu ist nötig die pädagogische Beobachtung «. Bei T. Fritzsch wie auch in den programmatischen Ausführungen von Ernst Meumann, der 1912 das Hamburger »Institut für Jugendforschung « mitbegründete, finden sich zudem Überlegungen, die pädagogischen Forschungsanstrengungen nicht auf das Feld der Schule zu begrenzen. In dem Hamburger Jugendinstitut ging es einerseits um die Initiierung von Untersuchungen zur »Entwicklung der Jugend selbst, sodann um das soziale Problem der Beziehungen der Jugendlichen zu den sozialen Verhältnissen, unter denen sie aufwachsen, und endlich um das Kulturproblem" sowie um die Frage, welche "Bedeutung eine rationelle Organisation der Jugendbildung für das geistige und wirtschaftliche Leben eines Volkes hat" (MEUMANN, 1912, S. 3). Für entsprechende Erkundungen beanspruchte das Hamburger Institut, eine "wissenschaftliche Instanz zu sein, die sich »vollkommen fernhält von aller Schulpolitik « und »die nichts erstrebt als systematische Erforschung des geistigen und sittlichen Lebens der Jugend und der Einflüsse, unter denen es tatsächlich steht (MEUMANN, 1912, S. 18). T. Fritzsch und E. Meumann, der allerdings nicht nur für den Einsatz von beobachtenden, sondern auch und insbesondere von statistisch-quantifizierenden Verfahren votiert, stehen zu Beginn des zurückliegenden Jahrhunderts exemplarisch für das Projekt, die Pädagogik für qualitative, vornehmlich auf systematische 
Beobachtungen sich stützende empirische Beobachtungen der heranwachsenden Generationen zu motivieren.

Ein quantitativ orientiertes Forschungsprogramm etabliert sich in breiterer Form in der Pädagogik erst sukzessive ab Beginn der 1930er und mit zunehmender Intensität dann ab Ende der 1950er Jahre im Zuge der realistische Wende (vgl. ROTH, 1963) in der deutschsprachigen pädagogischen Forschung. Bis heute entspricht ihre methodische Schrittfolge der Begriffsbildung, Operationalisierung und Messung in ihrer auf Systematik, Objektivität und instrumentelle Quantifizierung bedachten Methodik eher dem technizistischzweckrationalen Zeitgeist als die spurenorientierte Forschung (vgl. BONSS, 1982, S. 83). Im Gegenzug verliert in diesem Zeitraum die monographische, also im Kern qualitativ ausgerichtete Forschungstradition an Bedeutung, geriet aber immerhin nicht gänzlich in Vergessenheit. Im Kern existierten immer zwei Konzepte nebeneinander, das szientistische, statistische Programm als das offizielle und das spurensichernde als das inoffizielle.

In Reflexion dieser beiden forschungsmethodologischen Konzepte wird das quantitativ-statistische Paradigma als Tatsachenempirie und qualitativverstehende,rekonstruktivausgerichteteForschungsprogrammalsTotalitätsempirie diskutiert. Diese Markierung, die den quantitativen Verfahren vorhält, Kontexte und gesellschaftliche Zusammenhänge unbeachtet zu lassen, schließt an die Kritik der traditionellen Universitätsforschung von Max Horkheimer und insbesondere von Th. W. Adorno an, der gegenüber dem zahlenfixierten Forschungsparadigma einwendet, dass das Ziel der Entzauberung der Welt sich in dem quantifizierenden Programm reduziert auf die

[...] statistischen Aufbereitung [...] über mehr oder minder zahlreiche einzelne Personen [...]. Die Dinghaftigkeit der Methode, ihr eingeborenes Bestreben, Tatbestände festzunageln, wird auf ihre Gegenstände, eben die ermittelten subjektiven Tatbestände, übertragen, als ob dies Dinge an sich wären und nicht vielmehr verdinglicht. Die Methode droht sowohl ihre Sache zu fetischisieren wie selbst zum Fetisch zu entarten (ADORNO, 1962, S. 208).

Die sich in diesen Überlegungen kommunizierende Kritik an dem Methodenreservoir und den Deutungskompetenzen der klassischen, quantitativen Sozialforschung wie auch der pädagogischen Forschung wird zum inhaltlichen Orientierungspol der Einwände gegen die tatsachenzentrierte Sozialforschung und zum Votum für eine auf qualitative Daten basierende Totalitätsperspektive.

Der sehr verdichtete Rückblick weist erstens darauf hin, dass in der Pädagogik Forschung schon weit vor der sogenannten empirischen Wende im Kontext der pädagogischen Konzept- und Theoriebildung eine nicht unwesentliche Bedeutung spielt, allerdings bis zu Beginn der 1970er Jahre eher im Schatten der bis dahin 
dominanten geisteswissenschaftlichen Pädagogik. Die vorliegenden Studien belegen zweitens, dass die empirischen Bemühungen vor den 1970er Jahren sich nicht auf die eigentlichen Unterrichtsprozesse reduzieren, sich also keineswegs durchgängig als schulische Lehr-Lern-Forschung konzipierten. Die vorliegenden empirischen Studien illustrieren neben dieser Sensibilität für die Bildungsprozesse in den unterschiedlichsten sozialen und pädagogischen Arrangements und Räumen drittens eine beeindruckende methodische Vielfalt.

\section{Zum Stand der empirischen Bildungsforschung}

Die erziehungswissenschaftliche - respektive die pädagogische - Forschung gewann seit der Jahrtausendwende deutlich an Volumen und Bedeutung. Dass diese Lesart der Entwicklung der erziehungswissenschaftlichen Forschungskultur allgemein so nicht wahrgenommen wird, hat mehrere Gründe und findet eine Ursache in unterschiedlichen Problembereichen:

- Erstens scheint die Eigenständigkeit und Sichtbarkeit der pädagogischen, erziehungswissenschaftlichen Forschung im Kontext der internationalen Vergleichsstudien und Interdisziplinarität der empirischen Bildungsforschung unsichtbarer zu werden. Erziehungswissenschaftliche Forschungsvorhaben firmieren nicht mehr durchgehend und wahrscheinlich nur noch in einer Minderheit unter dem Etikett »Pädagogische Forschung «. Insbesondere die pädagogische Schulforschung und Unterrichtsforschung, aber auch Projekte, die sich beispielsweise nach den strukturellen Bedingungen des Aufwachsens von Kindern und Jugendlichen erkundigen, werden als Vorhaben der empirischen, auch soziologisch und psychologisch ausgerichteten Bildungsforschung ausgewiesen. Ob Forschungsvorhaben zu pädagogischen Fragen von der Soziologie, der Politikwissenschaft, der Psychologie oder eben von der Erziehungswissenschaft verantwortet werden, scheint keine besondere Relevanz mehr zu haben.

- Zweitens wird in der öffentlichen Wahrnehmung und auch weitgehend im forschungsbezogenen Diskurs die Eigenständigkeit der erziehungswissenschaftlichen Forschung nicht nur kaum noch thematisiert, sondern auf die schulische Lehr-Lernforschung und die schulbezogene Unterrichtsforschung reduziert. Forschungsmethodologische Diskurse werden verengt und beziehen sich häufig lediglich noch auf den Typus der schulbezogenen Forschung.

- Drittens wird in den bedeutenden Diskursen und Forschungsprogrammen die bestehend forschungsmethodische Vielfältigkeit im Rahmen der 
empirischen Bildungsforschung weitgehend identisch gesetzt mit Verfahren des quantitativ-heuristischen Forschungsspektrums. Die Bedeutung des qualitativ-rekonstruktiven Methodenfeldes wird parallel übersehen oder abgewertet beziehungsweise als »Spielwiese für die Generierung von subjektiven Daten« diskreditiert. Zudem werden historisch ausgerichtete oder erziehungsphilosophisch motivierte Forschungsvorhaben zuweilen nicht als empirische Projekte der Bildungsforschung angesehen.

- Und letztendlich viertens ist ein Auseinanderdriften zwischen den erziehungswissenschaftlichen-undauchsoziologischen-Theoriediskursenund den empiriebasierten Diskursen zu beobachten. Erziehungsphilosophischen, bildungs- und sozialisationstheoretischen, aber auch bildungs- und erziehungssoziologischen Erkenntnissen und Wissensbeständen wird in der Konzeptualisierung von empirischen Projekten nur noch eine Relevanz in Bezug auf den jeweils zu referierenden Forschungsstand, kaum jedoch noch bezüglich der theoretischen Rahmung von Forschungsvorhaben zugesprochen.

Einwände gegen eine deutlichere empirische Ausrichtung der Erziehungswissenschaft und der pädagogischen Diskussionen werden in der Regel mit Hinweisen auf diese Aspekte begründet. Demgegenüber ist an den Einwand von Th. W. Adorno zu erinnern. Mit Blick auf die theoretische, geisteswissenschaftliche Soziologie formuliert er, dass »angesichts der spezifischen Verflechtung sozialer Theoriebildung [...] ein Korrektiv, wie es die Researchmethoden anbieten«, zu entwickeln ist, denn "zahllose Behauptungen sozialer Theorien [...] können nur durch strenge Erhebungen überprüft und widerlegt werden" (ADORNO, 1962, S. 215). Und präzisierend fügt er an anderer Stelle hinzu:

Korrektiv ist die empirische Sozialforschung nicht nurinsofern, als sie blinde Konstruktionen von oben her verhindert, sondern auch im Verhältnis von Erscheinung und Wesen. Hat die Theorie der Gesellschaft den Erkenntniswert kritisch zu relativieren, so hat umgekehrt die empirische Forschung den Begriff des Wesensgesetzes vor Mythologisierung zu behüten (ADORNO, 1962, S. 220).

Pädagogisches, erziehungswissenschaftliches Denken kann sich heute nicht mehr ausschließlich auf die Neuinterpretation von klassischen Texten der Pädagogik konzentrieren und darauf vertrauen, so neues Wissen und Theorien über das Pädagogische zu gewinnen. Erziehungswissenschaftliche Reflexionen bedürfen einer Vergewisserung über empirisch generiertes Wissen. Und dieses Wissen, das wiederum einer theoretischen Rahmung bedarf, wird inzwischen unter Aktivierung von vielfältigen Forschungsmethoden gewonnen. ${ }^{1}$ 
1. Zunächst ist bezüglich der Verfahren und Designs, die Fragestellungen unter Bezug auf quantitative Methoden zu beantworten wünschen, festzuhalten, dass die Aussagekraft rein deskriptiv angelegter Studien inzwischen fast durchgängig kritisch diskutiert wird. Mehrebenenorientierte, diagnostische quer- und längsschnittbezogene Studien gewinnen an Bedeutung und Akzeptanz. Neben immer noch repräsentativ ausgerichteten Umfrage-, Querschnitts- und Panoramastudien ist eine methodische Qualifizierung bei der Durchführung von Panel- und Zeitwandelstudien auf der Basis von Mehrebenenmodellen zu erkennen. Im Kontext der Etablierung der erziehungswissenschaftlich wie auch soziologisch und psychologisch grundierten Bildungsforschung ist eine über theoretische Modelle abgesicherte Entwicklung wahrzunehmen, die sich insbesondere in der Durchführung der international wie national ausgerichteten Studien zur Erfassung von basalen, in schulischen Kontexten erworbenen Kompetenzen wie auch in Studien andeutet, die Ursache-Wirkungs-Beziehungen zu erfassen versuchen. Teilweise in Ergänzung hierzu, partiell mit den entsprechenden Studien eng verwoben sind zudem Bemühungen zu erkennen, über evidenzbasierte Designs die immer wieder thematisierte Differenz zwischen Forschung und Praxis klarer zu erfassen. Zudem ist die Wiederentdeckung von Verfahren zu erkennen, die, insbesondere in pädagogischen Kontexten, Programme testen und zudem diese zu implementieren versuchen. Darüber hinaus ist zu registrieren, dass in zunehmend mehr Forschungsvorhaben auf eine rein hypothesengeleitete und -prüfende Grundkonzeption verzichtet wird. Stattdessen werden anhand von theoretisch modellierten Modellen Fragestellungen entwickelt und empirisch überprüft (vgl. u. a. GRÄSEL, 2011).

Die Mehrzahl der pädagogisch relevanten Fragen lassen sich allerdings nicht mittels quantifizierender Verfahren aufklären, auch weil diese lediglich eingeschränkt gesellschaftlich und strukturelle Dimensionen berücksichtigen. Wissend, dass »alles gesellschaftlich Einzelne [...] die allgemeinen Bestimmungen in sich «(ADORNO, 1962, S. 212) tragen, gewannen qualitative Methoden insbesondere in der pädagogischen Forschung an Bedeutung. Mit den qualitativ gewonnenen Befunden liegen auch immer generelle Erkenntnisse über das Allgemeine vor. In Bezug auf die Erweiterung, Verfeinerung und theoretische Fundierung forschungsmethodischer Zugänge, Verfahren und methodologischer Präzisierungen sind vier Entwicklungen in Bezug auf qualitativ-verstehende Methoden besonders zu betonen. 
1. Die methodischen Verfahren, die in der erziehungs- und sozialwissenschaftlichen Forschung insbesondere mit dem Ziel eingesetzt wurden, über die Rekonstruktion narrativer Strukturen in autobiografischen Interviews Prozessstrukturen des Lebenslaufes erschließen zu können, sind weiterentwickelt worden. Neben Studien, die mittels biographischnarrativer Zugänge generationsübergreifende Entwicklungen und familiale Entwicklungen und Positionierungen aufzuklären suchen, ist insbesondere eine Entwicklung unter dem Stichwort „bildungstheoretische Biographieforschung“ zu erkennen (vgl. KOLLER; WULFTANGE, 2014).

2. Ein prominentes, zunehmend rezipiertes und umgesetztes Konzept der qualitativ-rekonstruktiven erziehungs- wie sozialwissenschaftlichen Forschung stellt inzwischen das auch im Anschluss an die Wissenssoziologie Karl Mannheims und die Ethnomethodologie Harold Garfinkels entwickelte Interpretationsverfahren der Dokumentarischen Methode dar. In erziehungs- und sozialwissenschaftlichen Forschungsprojekten werden mit diesem methodologischen Programm inzwischen eine Vielzahl von Fragestellungen mit unterschiedlichen Verfahren (Gruppendiskussionen, Interviews, Fotos, Videos) bearbeitet. Ziel ist dabei die Identifizierung von individuellen oder kollektiven Orientierungsmustern (vgl. BOHNSACK; PFAFF; WELLER, 2010).

3. Die Rekonstruktion von kulturellen und gesellschaftlichen Strukturen hat das von Ulrich Oevermann entwickelteVerfahren der strukturalen respektive Objektiven Hermeneutik zum Ziel. Dieses forschungsmethodische Spektrum ist in den letzten Jahrzehnten in einer Vielzahl von erziehungs- und sozialwissenschaftlichen Forschungsprojekten eingesetzt und mittlerweile nicht nur für die Auswertung von familialen Interaktionsprotokollen, sondern auch für die Analyse von Interviews, Fotos, Videos, Kunstwerken oder Internetseiten genutzt worden (vgl. OEVERMANN, 2004).

4. Im Rahmen der ethnografischen erziehungs- und sozialwissenschaftlichen Forschung haben sich im vergangenen Jahrzehnt neue Ansätze einer reflexiven Ethnografie herausgebildet. Diese gehen nicht mehr von der Vorstellung aus, dass man gestützt auf die Methoden der teilnehmenden Beobachtung ein abbildgetreues und einheitliches Bild von einer fremden Lebenswelt erstellen kann. Stattdessen betonen sie die soziale Konstruiertheit von ethnografischen Texten und weisen darauf hin, dass der Forschungsprozess ein permanenter Aushandlungsprozess ist. Unter dem Etikett der Ethnographie verbergen sich allerdings inzwischen unterschiedliche Konzepte - Ethnographie ist nicht gleich Ethnographie. 
Allgemein ist herauszustellen, dass das an die Ethnographie adressierte Frage- und Erkenntnisinteresse und das von der Ethnographie realisierte Forschungsprogramm sich im zurückliegenden Jahrzehnt deutlich erweitert und neu akzentuiert hat. Neben einer Ethnographie des "Wie etwas ist" hat sich eine Ethnographie des "Wie sich etwas herstellt und reproduziert" platziert. Ethnographie findet insbesondere auch in den organisations- und professionsorientierten Studien Anwendung. Aufgegriffen werden Ansätze einer Ethnographie des Pädagogischen im Kontext der allgemeinen pädagogischen Organisationsforschung - hier insbesondere bezogen auf die Praxen in der Erwachsenenbildung und der Fort- und Weiterbildung - und in den Recherchen zum Umgang mit Wissen (vgl. HEINZEL et al., 2010).

In besonderer Form hat sich in den zurückliegenden Jahrzehnten auch die erziehungswissenschaftliche, historisch ausgerichtete Erziehungs-, Sozial-, Bildungs- und Kulturforschung entwickelt und so neue Aufmerksamkeit gefunden:

1. Im Schatten der Herausbildung und Etablierung der historischen Kulturforschung verfeinerte und spezialisierte sich auch die erziehungswissenschaftliche historische Forschung und erweiterte zudem ihren Aufmerksamkeitsfokus auch in Bezug auf die letzten Jahrzehnte des zurückliegenden Jahrtausends. Keineswegs neu, aber neu akzentuiert wird in jüngerer Zeit wieder vermehrt auf Befunde der Geschichtswissenschaft rekurriert. Insgesamt verliert die historische Sozial- und Bildungsforschung ihre Textgebundenheit und neue Formen der Kontextualisierung, aber insbesondere neue forschungsmethodische, sprach- und diskursanalytische Methoden finden mehr Zuspruch (vgl. REH et al., 2016).

Weiterhin sind Fortschritte in der Erziehungswissenschaft und Soziologie auch im Hinblick auf die Frage der Triangulation - mixed methods - von Daten wahrzunehmen. Waren die methodologischen Diskussionen in den 1970er und 1980er Jahren noch durch heftige wissenschaftstheoretische Kontroversen zwischen den Repräsentanten qualitativ-rekonstruktiver und quantitativ-statistischer Forschungsansätze bestimmt, so plädieren Vertreter beider Richtungen inzwischen dafür, qualitative und quantitative methodische Zugriffe zu kombinieren.

Insgesamt ist wahrzunehmen, dass die bestehenden methodischen und theoretischen, aber auch forschungsethischen Differenzen zwischen unterschiedlichen Theorieschulen, insbesondere aber zwischen quantitativ-statistischen und qualitativ-rekonstruktiven Verfahren nicht mehr durchgängig scharf markiert werden. Zunehmend deutlicher wird darauf verwiesen, dass die divergierenden 
forschungsmethodologischen Zugänge und Annahmen oftmals in Bezug auf die Generierung und forschungsmethodische Operationalisierung von Fragestellungen mit vergleichbaren Herausforderungen konfrontiert sind. Obgleich ein Ende der Diskussionen um die Adäquatheit qualitativer und quantitativer Methoden noch nicht gänzlich beendet scheint, der Methodenstreit fortdauert, ist abzusehen, dass es zukünftig nicht mehr vornehmlich um die weitere Abgrenzungen der unterschiedlichen Forschungsstrategien gehen wird, sondern darum, die gewählte methodische Orientierung zu präzisieren und ihre Angemessenheit für die gewählten Fragestellungen zu belegen. Dies bedeutet auch, auf die Kennzeichnung quantitativ generierter Daten als "objektiv" und qualitativ erhobener Daten als "subjektiv" zu verzichten. Im Kern zielen beide methodischen Forschungsmilieus darauf ab, der "gesellschaftlichen Konstruktion von Wirklichkeit" (BERGER; LUCKMANN, 1980) nachzuspüren. Zumindest eine kritische, gesellschaftsanalytisch grundierte empirische pädagogische Bildungsforschung wird keine klugen Argumente finden, hinter dieser konzeptionellen Prämisse erziehungswissenschaftlicher Forschung zurückzufallen, es sei denn, sie befriedigt sich mit der Rolle affirmativer Akklamationen. Damit wird der Idee entsprochen, dass der »Gegensatz zwischen quantitativer und qualitativer Analyse nicht absolut ist«(ADORNO, 1962, S. 212).

\section{Erziehungswissenschaftliche Forschung - Ausblick}

Die Überlegungen in diesem Beitrag plädieren für ein offenes, zumindest erweitertes Verständnis von empirischer pädagogischer Bildungsforschung. Das in diesem Aufsatz vorgestellte Programm einer pädagogischen Forschung

- umfasst sowohl quantitativ-statistische wie auch qualitativ-verstehende und historisch ausgerichtete forschungsmethodologische Konzepte und methodische Verfahren,

- sieht von der Pädagogik der Kindheit über die schulischen und sozialpädagogischen Handlungsfelder, die Erwachsenenbildung bis hin zur Pädagogik des Alters und des Alterns als Forschungsfelder an und

- zeigt sich distanziert gegenüber Unternehmungen, zwischen empirischer Forschung und erziehungswissenschaftlicher und -philosophischer Theoriebildung Grenzen zu markieren.

In dem Beitrag wird keineswegs für eine pädagogische Bildungsforschung plädiert, dielediglich dieEffektevon Bildungsprozessen dokumentiert.Pädagogische Forschung, so wird argumentiert, bleibt herausgefordert, die Strukturen, Modalitäten, Rahmenbedingungen, Zugänge und Teilhabemöglichkeiten wie auch die Praxen und Konstitutionsbedingungen von pädagogischen Handlungsszenarien aufzuklären. Und dazu bedarf es auch der theoretischen Reflexion des empirisch 
gewonnenen Wissens, denn der Theorie fällt die Aufgabe $\mathrm{zu}, \mathrm{zu}$ benennen, "was insgeheim das Getriebe zusammenhält" (ADORNO, 1962, S. 205). Die Theorie schützt davor, auf Basis von vermeintlichen Fakten die gesellschaftliche Wirklichkeit lediglich zu reproduzieren. Entkoppelt sich die Theorie jedoch von den empirischen Befunden, unterliegt sie der Gefahr, lediglich Ideologien zu produzieren. "Empirie und Theorie lassen sich nicht in ein Kontinuum eintragen" (ADORNO, 1962, S. 207), sind aber unabhängig voneinander nicht denkbar, wenn sie jeweils mehr zu sein wünschen, als Instrumente der Konsolidierung des Bestehenden und der hervorgebrachten Lebensformen in der kapitalistischen Moderne (vgl. JAEGGI, 2014).

Ein Forschungsverständnis, das diese Prämissen akzeptiert, ist gefeint davor, die empirische Forschung gegen die Philosophie der Erziehung oder die kritische Bildungsphilosophie gegen empirisch grundierte Erkenntnisintentionen zu positionieren. Pädagogische Theoriebildung ist heute nicht mehr ohne empirisch generiertes Wissen möglich wie andersherum empirische Datengewinnung nicht verzichten kann auf Theorie.

\section{Note}

1 Die Anmerkungen zum Stand der empirischen Forschung basieren auf einen gemeinsam mit H. H. Krüger publizierten Beitrag an (KRÜGER; THOLE, 2015).

\section{Literatur}

ADORNO, Th. W. Soziologie und empirische Forschung. In: HORKHEIMER, M.; ADORNO, TH. W. Sociologica II. Frankfurt am Main: Suhrkamp Verlag., 1962. S. 205-222.

. Theorie der Halbbildung. In: HORKHEIMER, M.; ADORNO, TH. W. Sociologica II. Frankfurt am Main: Suhrkamp, 1973. S. 168-191.

BENNER, D. Allgemeine Pädagogik. Weinheim u. Basel: Juventa, 1987.

BENNER, D.; BRÜGGEN, F. Bildsamkeit/Bildung. In: BENNER, D.; OELKERS, J. (Hrsg.). Historisches Wörterbuch der Pädagogik. Weinheim u. Basel: Juventa, 2004. S. 174-215.

BERGER, P. L.; LUCKMANN, T. Die gesellschaftliche Konstruktion der Wirklichkeit. Frankfurt am Main: Fischer, 1980.

BERNFELD, S. Sisyphos oder die Grenzen der Erziehung. Frankfurt am Main: Suhrkamp Taschenbuch Wissenschaft, 1973.

BOHNSACK, R.; PFAFF, N.; WELLER, W. Qualitative Analysis and Documentary Method in International Educational Research. Opladen u. Farmington Hill: Barbara, 2010.

BONSS, W. Die Einübung des Tatsachenblicks. Frankfurt am Main: Suhrkamp, 1982.

BOURDIEU, P. Le misère du monde. Paris: Seuil, 1993.

. Sozialer Raum und Klassen. Frankfurt am Main: Suhrkamp, 1985. 
EHRENSPECK, Y. Bildung. In: KRÜGER, H. H.; GRUNERT, C. (Hrsg). Wörterbuch Erziehungswissenschaft. Wiesbaden: Verlag für Sozialwissenschaften, 2004. S. 64-71.

FRITZSCH, T. Zur Geschichte der Kinderforschung und Kinderbeobachtung. Zeitschrift für Philosophie und Pädagogik, 13. Jg. 1906, S. 497-506.

GRÄSEL, C. Was ist empirische Bildungsforschung? In: REINDERS, H.; DITTON, H.; GRÄSEL, C.; GNIEWOSZ, B. (Hrsg.). Empirische Bildungsforschung. Strukturen und Methoden. Wiesbaden: Verlag für Sozialwissenschaften, 2011. S. 13-27.

HEINZEL, F. et al. (Hrsg.). "Auf unsicherem Terrain”. Ethnographische Forschung im Kontext des Bildungs- und Sozialwesens. Wiesbaden: Verlag für Sozialwissenschaften, 2010.

HEYDORN, H.-J. Bildungstheoretische und pädagogische Schriften. Bd. 4. Vaduz: Topos, 1995.

JAEGGI, R. Kritik der Lebensformen. Frankfurt am Main: Suhrkamp, 2014.

KOLLER, H.-CH.; WULFTANGE, G. (Hrsg.). Lebensgeschichte als Bildungsprozess. Bielefeld: Transcript, 2014.

KRÜGER, H.-H.; THOLE, W. Forschung als Herausforderung - Einleitung. Entwicklung der Promotionsförderung in der Erziehungswissenschaft und den Sozialwissenschaften - Hinweise und Anmerkungen. In: KRÜGER, H. H.; SÜNKER, H.; THOLE, W. (Hrsg.). Forschung als Herausforderung. Methodologische Ansprüche und Praxis in erziehungsund sozialwissenschaftlichen Projekten. Berlin u. Toronto: Barbara Budrich, 2015. S. 11-22.

MEUMANN, E. Über Institute für Jugendkunde. Leipzig u. Berlin: [s. n.], 1912.

MOSE. In: LUTHERS M. Bibel. Die Heilige Schrift nach der Deutschen Übersetzung. K. 1, v. 26, [s.n.], 1912.

OEVERMANN, U. Die elementare Problematik der Datenlage in der quantifizierenden Bildungs- und Sozialforschung. In: Sozialer Sinn, 5. Jg. 2004, Heft 3, S. 413-475.

REH, S. et al. Wissen machen. Zeitschrift für Pädagogik. Beiheft 63. Weinheim u. Basel, 2016.

ROTH, H. Die realistische Wendung in der pädagogischen Forschung. Die deutsche Schule, 55. Jg., Heft 3, 1963, S. 109-119.

STOJANOV, K. Bildung und Anerkennung. Wiesbaden: VS-Verlag, 2006.

THOLE, W. Forschung, Theoriebildung und pädagogisches Handeln. Reflexionen zum Verhältnis von Theorie und Praxis im Zeitalter der "Entgrenzung des Pädagogischen". In: GRUNERT, C.; VON WENSIERSKI, H.-J. (Hrsg.). Jugend und Bildung. Modernisierungsprozesse und Strukturwandel von Erziehung und Bildung am Beginn des 21. Jahrhunderts. Opladen u. Farmington Hills: Budrich, 2008. S. 79-99.

TIPPELT,R.BildungundsozialerWandel.EineUntersuchungvonModernisierungsprozessen in der Bundesrepublik Deutschland seit 1950. Weinheim: Deutscher Studien, 1990.

ZIRFAS, J. Bildung. In:KADE, J. u. a.(Hrsg).Pädagogisches Wissen.Erziehungswissenschaft in Grundbegriffen. Ein Grundkurs in 30 Stichworten. Stuttgart: Kohlhammer, 2010. S. 13-20. 